\title{
Neurodevelopment and the Origins of Brain Disorders
}

\author{
Neuropsychopharmacology Reviews (2015) 40, I-3; doi: I 0. I 038/npp.20 I4.237
}

A ccumulating data from epidemiology, genetics, epigenetics, basic neuroscience, and neuroimaging point to neurodevelopment as a time for establishing risk and resilience to later neuropsychiatric illness. The historical divide between childhood neurodevelopmental disorders and disabilities and adult mental illness is increasingly recognized as artificial. Since the time of Waddington's formulation of an epigenetic landscape in which heritable and external factors modulate development to set the stage for later function, correlative findings have fit the model of a neurodevelopmental basis for mental illness. Recent technical and experimental advances have generated a more mechanistic understanding of how perturbations of brain development can result in either the early or late appearance of constellations of behavioral and cognitive symptoms that define categorical illnesses. The field has now determined some factors that influence neurodevelopment during sensitive and critical periods, specific times when environmental input combines with genetic programming to influence brain patterning, neuronal diversity, connectivity, and, ultimately, functional maturation. The field remains an exciting work in progress, fueled by a desire to move from demonstration of correlation to understanding of mechanistic causation and then, ultimately, to prevention or early intervention that harnesses brain plasticity. The initial emphasis on limited, critical time periods is now changing through interdisciplinary research that has redefined adaptive capacities in animals and in humans through treatments and interventions.

Our goal in organizing this issue of Neuropsychopharmacology Reviews was for disciplinary experts to provide both experimental highlights and perspectives on the current understanding of basic mechanisms of neurodevelopment, the influence of developmental disruptions on childhood and adult-onset brain and behavioral disorders, and the emerging promise of translation into treatments. We recognize that there is insufficient space here to cover all of the relevant literature on neurodevelopment and mental illness. Our hope is that this issue will generate curiosity and harness enthusiasm, thereby stimulating further research toward a mechanistic understanding of neurodevelopment in the context of mental illness and neurodevelopmental disorders.

The issue begins with four articles on basic principles of neurodevelopment with relevance to the origins of disorders and disabilities. Washbourne (2015) provides a neurobiological roadmap for the molecular basis of synapse formation, which has come into focus as a target of disruption in disorders ranging from autism to schizophrenia. Specific gene families, implicated from the discovery of rare, causal mutations, are highlighted, as well as the ways in which genetic data may be useful for improving future diagnostic and treatment strategies. Chu and Anderson (2015) provide a basic framework for understanding the origins and development of neocortical interneurons. This topic is of particular importance because of the role of excitatory:inhibitory balance in cortical information processing, and its implication in many neurodevelopmental and psychiatric disorders. Hammock (2015) describes the development of neuropeptide systems, with a particular emphasis on oxytocin and vasopressin. A large adult literature has examined the role of these neuropeptides in influencing social cognition, maternal behavior and aggression, but more recent studies reveal a critical importance for the oxytocin and vasopressin systems during neurodevelopment, potentially pointing to opportunities for intervention. Giedd et al, (2015) review an increasing body of longitudinal neuroimaging studies that identify the developmental trajectories of brain regions and circuits, with an emphasis on understanding the relationships between maturing brain structure, function, and cognition. These studies provide some of the keys to decipher the critical puzzle of inter-individual variability.

The next four articles serve as a bridge, providing clinically relevant examples of basic developmental principles. Hartley and Lee (2015) provide an overview of sensitive developmental periods related to affective behavior. They describe mechanistic parallels between the development of fear circuitry and our classic understanding of sensitive periods in the development of primary sensory systems. Ross et al, (2015) focus on the pervasive issue of prenatal exposure to drugs of abuse, providing a neurobiological framework based upon basic research on the developmental impact on different cell populations and brain systems. They weave in a discussion of clinical outcomes and disorder risk in longitudinal human studies, emphasizing the importance of bridging the basic-clinical gap to yield new interventions. Suri et al, (2015) provide an advanced understanding of the genetic and early life experiences that influence monoamine systems during sensitive periods of development. With the popularity of 
these systems as therapeutic targets, they detail the intersections between mechanistic insights from preclinical research and translational relevance from emerging clinical research. Schoenfeld and Cameron (2015) tackle the challenges of understanding how perturbations in adult neurogenesis may contribute to neuropsychiatric disorders and their treatment. Although considerable literature points to the relevance of adult neurogenesis, particularly for depression, they emphasize the need for further research to test causation directly and to dissect underlying mechanisms.

As outlined in the next three articles, the environment has complex biological and behavioral influences across development that impact later risk for illness. Toth (2015) reviews recent, intriguing research on intergenerational, non-genetic transmission of epigenetic information that alters brain development and impacts risk for mental illness. He presents the current evidence for the impact of the prenatal environment on such transmission, delineates the limitations of this work, and maps out the potential for future studies in animal models and in humans. Kundakovic and Champagne (2015) focus on postnatal factors that impact epigenetic status to drive brain and behavioral development, drawing from precise experiments in rodent models. Potential molecular targets are highlighted, together with epigenetic biomarkers that may foreshadow risk of psychopathology later in life. The next article pivots to human studies on the effects of inadequate or abusive care during early childhood. Humphries and Zeanah (2015) examine the connections between resulting alterations in neurobiological development and ongoing risk for social dysfunction, attention-deficit hyperactivity disorder, and antisocial behavior.

Two disorder-specific reviews are presented in the next section. Hernandez et al, 2015 review the neurobiological signatures that have emerged from a decade of intensive genetic and neuroimaging research on children and adolescents with autism spectrum disorders. The discussion highlights the many challenges to understanding heterogeneous findings in the context of individual differences between children, as well as the technical caveats of current neuroimaging and analytical methodologies. Schmidt and Mirnics (2015) provide a practical evaluation of the evidence supporting the neurodevelopmental hypothesis of schizophrenia. The review integrates genetic risk factors that point, in part, to the development of interneurons, with a perspective on excitatory-inhibitory balance in the context of schizophrenia risk and pathophysiology.

The final two articles incorporate neurodevelopmental principles and an improving understanding of disorder etiology to discuss the promise of next-generation treatments. Henderson et al, (2015) focus on pediatric anxiety disorders, emphasizing emerging research that points to dysfunction in specific circuits. In pivoting to current and future strategies for treatment, both behavioral and pharmacological, they emphasize interventions that are similarly targeted to specific circuits. VeenstraVanderWeele and Warren (2015) review prevention or intervention strategies that connect to the developmental origins of neuropsychiatric disorders, touching on many of the areas covered in this special issue. Noting that existing interventions have typically not made use of mechanistic knowledge, they highlight the potential for new, targeted treatments, initially drawn from discoveries in animal models of syndromal disorders that include prominent neuropsychiatric symptoms.

By moving from basic developmental neurobiology, to mechanisms of neurodevelopmental risk, and finally to possible interventions drawn from these mechanistic insights, we hope to convince the reader of the critical importance of developmental neuroscience for the future of neuropsychopharmacology. Throughout this special issue, leaders in the field have woven clinical relevance into each review, whether their own research is focused on primarily basic mechanisms in model systems or exclusively on risk and resilience in humans. The cutting-edge science in these reviews is complemented by the exciting Hot Topics articles that complete the 2014 Neuropsychopharmacology Reviews issue.

\section{FUNDING AND DISCLOSURE}

PL is on the Scientific Advisory Board of Pediatric Biosciences. Jeremy Veenstra-VanderWeele has consulted with SynapDx, Roche Pharmaceuticals, and Novartis, and his research program has received funding for research from SynapDx, Roche Pharmaceuticals, Novartis, Forest, and Sunovion.

\section{ACKNOWLEDGEMENTS}

Our work as volume editors would not have been possible without the guidance and insight of Neuropsychopharmacology leadership, including Gwenn Smith, John Neumaier, and William Carlezon. We are also profoundly grateful for the editorial assistance of Natalie Marler, whose diligence and attention to detail made this issue possible.

Work in PL's research program is supported by grants from the NIH and the Simons Foundation. Work in JV's research program is supported by grants from the NIH, HRSA, and Autism Speaks.

\section{REFERENCES}

Washbourne P (2015). Synapse assembly and neurodevelopmental disorders. Neuropsychopharmacology 40: 4-15.

Chu J, Anderson SA (2015). Development of cortical interneurons. Neuropsychopharmacology 40: 16-23.

Hammock EAD (2015). Developmental perspectives on oxytocin and vasopressin Neuropsychopharmacology 40: 24-42.

Giedd JN, Raznahan A, Alexander-Bloch A, Schmitt E, Gogtay N, Rapoport JL (2015). Child psychiatry branch of the national institute of mental health longitudinal structural magnetic resonance imaging study of human brain development. Neuropsychopharmacology 40: 43-49.

Hartley CA, Lee FS (2015). Sensitive periods in affective development: nonlinear maturation of fear learning. Neuropsychopharmacology 40: 50-60.

Ross EJ, Graham DL, Money KM, Stanwood GD (2015). Developmental consequences of fetal exposure to drugs: what we know and what we still must learn. Neuropsychopharmacology 40: 61-87. 
Suri D, Teixeira CM, Cagliostro MKC, Mahadevia D, Ansorge MS (2015). Monoamine-sensitive developmental periods impacting adult emotional and cognitive behaviors. Neuropsychopharmacology 40: 88-112.

Schoenfeld TJ, Cameron HA (2015). Adult neurogenesis and mental illness. Neuropsychopharmacology 40: 113-128.

Toth M (2015). Mechanisms of non-genetic inheritance and psychiatric disorders. Neuropsychopharmacology 40: 129-140.

Kundakovic M, Champagne FA (2015). Early life experience, epigenetics, and the developing brain. Neuropsychopharmacology 40: 141-153.

Humphreys $\mathrm{KL}$, Zeanah $\mathrm{CH}$ (2015). Deviations from the expectable environment in early childhood and emerging psychopathology. Neuropsychopharmacology 40: 154-170.

Hernandez LM, Rudie JD, Green SA, Bookheimer S, Dapretto M (2015). Neural signatures of autism spectrum disorders: insights into brain network dynamics. Neuropsychopharmacology 40: 171-189.

Schmidt MJ, Mirnics K (2015). Neurodevelopment, GABA system dysfunction, and schizophrenia. Neuropsychopharmacology 40: 190-206.
Henderson HA, Pine DS, Fox NA (2015). Behavioral Inhibition and developmental risk: a dual-processing perspective. Neuropsychopharmacology 40: 207-224

Veenstra-VanderWeele J, Warren Z (2015). Intervention in the context of development: pathways toward new treatments. Neuropsychopharmacology 40: 225-237.

Pat Levitt ${ }^{1}$ and Jeremy Veenstra-VanderWeele ${ }^{2}$

${ }^{1}$ Developmental Neurogenetics, Institute for the Developing Mind Children's Hospital Los Angeles, Keck School of

Medicine, University of Southern California, University of Southern California Neuroscience Graduate Program, Los Angeles, CA, USA; ${ }^{2}$ New York State Psychiatric Institute,

Columbia University, New York, USA

E-mails: plevitt@med.usc.edu; jeremy.vvw@gmail.com 